\title{
'Being there' for women with metastatic breast cancer: a pan-European patient survey
}

\author{
V Harding ${ }^{1}$, M Afshar ${ }^{2}$, J Krell ${ }^{1}$, R Ramaswami ${ }^{1}$, C J Twelves ${ }^{2,3,4}$ and J Stebbing ${ }^{\star}, 1,4$ \\ ${ }^{1}$ Department of Medical Oncology, Imperial College Healthcare NHS Trust, 1st Floor, E Wing, Fulham Palace Road, \\ London, W6 8RF, UK; ${ }^{2}$ St James's Institute of Oncology, The Leeds Teaching Hospitals, Leeds LS9 7TF, UK and ${ }^{3}$ Leeds Institute \\ of Cancer Studies and Pathology, University of Leeds, Leeds LS9 7TF, UK
}

Background: Understanding their experiences of diagnosis is integral to improving the quality of care for women living with advanced/metastatic breast cancer.

Methods: A survey, initiated in March 2011, was conducted in two stages. First, the views of 47 breast cancer-related patient groups in eight European countries were sought on standards of breast cancer care and unmet needs of patients. Findings were used to develop a patient-centric survey to capture personal experiences of advanced breast cancer to determine insights into the 'trade-off' between extending overall survival and side effects associated with its treatment. The second online survey was open to women with locally advanced or metastatic breast cancer, or their carers, and responders were recruited through local patient groups. Data were collected via anonymous local language questionnaires.

Results: The online stage II survey received a total of 230 responses from 17 European countries: 94\% of respondents had locally advanced or metastatic breast cancer and $6 \%$ were adult carers. Although the overall experience of care was generally good/ excellent (77\%), gaps were still perceived in terms of treatment choice and information provision. Treatment choice for patients was felt to be lacking by $32 \%$ of responders. In addition, $68 \%$ of those who responded would have liked more information about future medical treatments and research, with $57 \%$ wishing to receive this information from their oncologist. Two-thirds (66\%) of women with advanced breast cancer, or their carers, believed life-extending treatment to be important so that they can spend more time with family and friends, and $67 \%$ said that the treatment was worthwhile, despite potential associated side effects.

Conclusion: These findings show a continuing need to provide women with advanced breast cancer with better information and emphasise the importance that these patients often place on prolonging survival.

Breast cancer is the most common cancer in women in most countries and the leading cause of cancer-related death in women worldwide. In 2008, there were an estimated 421000 new cases of breast cancer diagnosed in Europe (Ferlay et al, 2010) and 1.4 million worldwide (Ferlay et al, 2008). The incidence of breast cancer worldwide is predicted to rise to 2.3 million by 2030 , although mortality rates in Western countries are falling because of earlier detection and effective adjuvant treatment (Ferlay et al, 2008). Nevertheless, for the $4-6 \%$ of women with metastatic disease at diagnosis (Cardoso et al, 2011), and the approximately $30 \%$ who present with early breast cancer but later develop metastatic breast cancer (Shaughnessy, 2005), treatment effectively remains palliative. Although the median survival from diagnosis of metastatic disease is $2-3$ years, in indolent disease, it may be as long as 10-15 years (Johnston and Swanton, 2006). Hence, as the prevalence of advanced breast cancer increases, and overall survival is slowly extended, the needs of women living with metastatic disease continue to pose a major challenge for healthcare professionals.

The specific aims of treatment for patients with metastatic and locally advanced breast cancer vary depending on their presentation, symptoms and circumstances, but most patients and

${ }^{\star}$ Correspondence: Professor J Stebbing; E-mail: j.stebbing@imperial.ac.uk
${ }^{4}$ These authors contributed equally to this work.

Received 15 May 2013; revised 26 July 2013; accepted 30 July 2013; published online 3 September 2013

(C) 2013 Cancer Research UK. All rights reserved 0007-0920/13 
clinicians have a common goal of alleviating symptoms, maintaining quality of life and prolonging survival. Treatment options may include not only approved and generally available local and systemic therapies but also the possibility of participation in clinical trials. Factors that influence clinicians in treatment strategies include the molecular phenotype of the cancer, the site and extent of metastatic disease, the fitness of the patient, their prior therapy and any potential complications related to that treatment, as well as the patient's own preferences (Chung and Carlson, 2003). Providing adequate information for patients to be actively involved in decision making is an important role for healthcare professionals.

How patients use the information offered to them will undoubtedly vary, but we believe that many women living with advanced breast cancer share similar needs and opinions that are specific to their disease. The literature reports that information needs are high in patients with metastatic breast cancer (Mayer, 2010) and that those who have their needs me express much less anxiety and lower likelihood of depression (Mayer and Grober, 2006). Despite this evidence, patients living with advanced breast cancer continue to encounter challenges in accessing the required information and support. Indeed $90 \%$ of patients diagnosed with advanced breast cancer questioned in one study reported that they received less attention and less information than at the time of their initial diagnosis (Cella et al, 1990) and other studies have reported that the majority of patients found the diagnosis of recurrence to be more distressing than the original diagnosis (Cella et al, 1990; Mahon et al, 1990). Learning what patients want from their healthcare providers is an important step in addressing their concerns and meeting their psychosocial needs.

Several studies have looked at how patients with cancer (although not specifically breast cancer) view the relative importance of improved survival as opposed to greater treatment toxicity. Some have suggested that patients would accept considerable toxicity for very modest improvements in survival (Wong et al, 2012), whereas others have suggested that an improvement in survival alone is not sufficient in the trade-off against treatment-related toxicities (Bridges et al, 2012). There is, however, relatively little information specifically in the context of metastatic breast cancer. Interestingly though, a study in women with early breast cancer indicated that $76 \%$ would choose alleviation of symptoms rather than prolonged survival in a hypothetical scenario of metastatic disease (McQuellon et al, 1995). To date, there is very little understanding of whether women living with metastatic breast cancer are willing to tolerate treatmentrelated toxicities in order to extend their life, or what information and support they would like to help them to decide. Of course, the distinction between progression-free and overall survival may be a difficult concept to grasp when contemplating further aggressive treatments (or on deciding to have better tolerated therapies or no active treatment), and explanation and clarification are central to helping patients make such decisions.

In order to provide the best care for patients living with advanced breast cancer, we need to know more about their experiences of diagnosis, treatment and care. For the information to be useful, transferable and as widely applicable as possible, we undertook a two-part, pan-European survey of patients (or their carers) living with metastatic/advanced breast cancer. This is the first European-wide study to be conducted specifically aimed at gaining a better understanding of the needs of these patients. As the prevalence of advanced breast cancer increases because of increasing incidence and reducing mortality, this information will become increasingly more relevant in both developed and developing countries. The information obtained from this panEuropean study should empower patients, their carers, clinicians and managers to improve lines of communication and restructure services to address the needs of this patient group.

\section{MATERIALS AND METHODS}

The survey, initiated in March 2011, was conducted using a two-stage methodology developed in conjunction with academic and patient organisations. This methodology has been validated as an effective means of measuring patients' perspectives in healthcare systems (Wyke et al, 2008). The two-stage design was intended to overcome the limitations inherent in existing methodology; namely that questionnaires and surveys are typically constructed without first exploring patients' own opinions on the subject in question.

In 2011, the first stage of the 'Being There' survey was conducted among 47 patient groups based in eight European countries, all of which had links with patients living with locally advanced or metastatic breast cancer. Their views on standards of care and unmet needs of patients with metastatic and locally advanced breast cancer were sought through 14 open-ended questions allowing the groups to respond as comprehensively as they wished. Responses were collated from Belgium, France, Germany, Italy, Sweden, Switzerland and the United Kingdom. The results were used to design the survey for stage II by formulating the patient group statements into a multiple-choice questionnaire, from which respondents were asked to specify their priorities from a list of options.

The second stage was planned to identify the important issues faced by women with locally advanced and metastatic breast cancer in an online survey (Supplementary Appendix 1) open to women aged 20 years or older (no upper age limit) with locally advanced or metastatic breast cancer, or their carers, at any stage in their treatment. Responders were recruited through local patient groups, and data were collected via anonymous local language questionnaires. The translations were performed in accordance with current good practice in translation of international survey questionnaires. The English master version was subject to parallel translation into the language of choice by independent translators familiar with survey research. 'Back translation' was conducted to check validity of the terminology and the translated version was pretested in the participating country. The questionnaire explored topics including patients' personal experiences of diagnosis and treatment, the attitudes of health professionals within their cancer services, the information provided and made available and patients' views on improving the future care offered. The study also sought to gain insight into the 'trade-off between extending overall survival and treatment-related side effects. PatientView, an independent research-and-publishing organisation, who were involved in the design of the questionnaire, performed the analyses.

\section{RESULTS}

A wide variety of patient support groups were contacted, all of whom had direct contact with patients with advanced breast cancer. Fifty-eight percent of these groups cited breast cancer as their organisation's main 'specialisation', others stated palliative care, bereavement, carers and women's health as their main provision of support (Supplementary Appendix 2). Groups from Belgium(3), France(14), Germany(13), Italy(22), Spain(9), Sweden(23), Switzerland(3) and the United Kingdom(18) took part. The responses given were varied but often repeated by other groups from other countries. The most frequently repeated answers from stage I are shown graphically in a series of charts in Supplementary Appendix 2.

In Stage II of the study, a total of 230 responses were received from 17 European countries; 216 responders (94\%) had locally advanced or metastatic breast cancer and $14(6 \%)$ were adult carers (Table 1). The details of the online survey were distributed by patient support groups and hence the number of people deciding 
Table 1. Demographics of patient/carer responders to stage ॥

\begin{tabular}{|l|c|}
\hline Age (years) & $\%$ \\
\hline Under 20 & 0 \\
$20-29$ & 2 \\
$30-39$ & 7 \\
$40-49$ & 32 \\
$50-59$ & 33 \\
$60-69$ & 23 \\
$70-79$ & 1 \\
80 or older & 2 \\
\hline Breast cancer patient & 94 \\
\hline Carer & 6 (all female) \\
\hline Country of residence & \\
\hline Cyprus & 2 \\
Czech Republic & 2 \\
Denmark & 4 \\
Greece & 2 \\
Hungary & 16 \\
Iceland & 1 \\
Ireland & 3 \\
Lithuania & 1 \\
Poland & 1 \\
Romania & 1 \\
Sweden & 7 \\
United Kingdom & 47 \\
Other & 7 \\
Unanswered & 6 \\
\hline
\end{tabular}

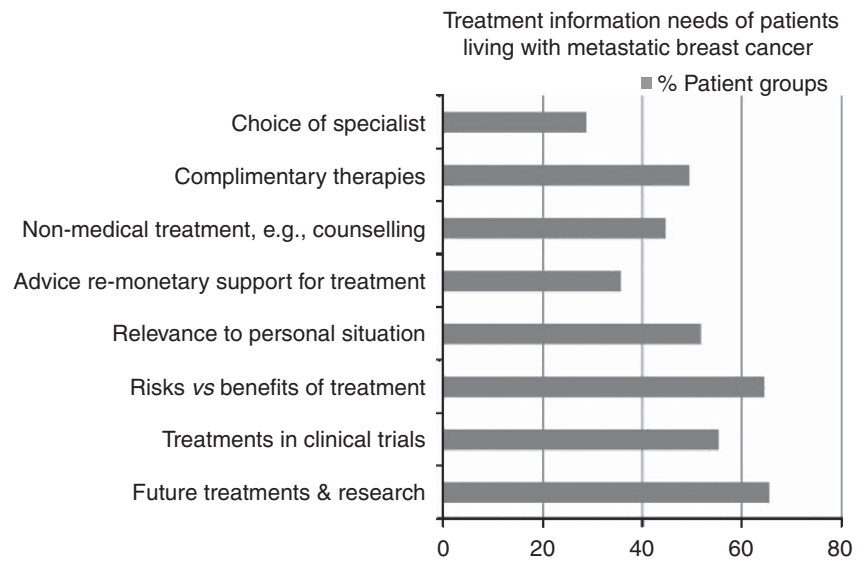

Figure 1. Treatment information needs in metastatic breast cancer. What further related information is required.

not to take the survey is unknown. Most responders (77\%) rated their care as good to excellent, but clear gaps were evident in both the treatment choice and information provision for these patients.

Treatment choice. A third (32\%) of patients/carers perceived treatment choice within their country's healthcare system to be lacking. The majority (59\%) felt they had choice in the treatment decisions, and only $21 \%$ had been informed of treatments available through clinical trials. Nevertheless, half (56\%) reported that they were not advised about treatment options available outside of their hospital.

Information provision. Patients/carers need more information on treatment-related issues (Figure 1). Two-thirds (68\%) of responders wanted more information about future medical treatments and research; the majority of patients/carers felt that this information should come from their oncologist. Half (53\%) of patients/carers wanted to receive information on treatments currently in clinical trials and the same proportion (55\%) wanted more information on the balance between the benefits of treatment and the risks.

Quality of care. Important areas were identified where healthcare professionals could improve the quality-of-care provision to women with locally advanced or metastatic breast cancer (Figure 2). About half of patients/carers felt their care would be enhanced if healthcare professionals listened more to patients (51\%); ensured continuity of care with the same doctors, nurses and specialists (55\%); reduced waiting times for access to treatment (52\%); and ensured counselling was available if required (55\%).

Life-extending treatments. Survival was the priority for the majority of women with locally advanced or metastatic breast cancer, patients being willing to 'trade-off drug side effects in order to prolong their life (Figure 3). Two-thirds (67\%) of patients/ carers believed life-extending treatment to be important in order to extend time spent with family and friends, and two-thirds (64\%) judged treatment worthwhile if it prolonged survival, irrespective of potential side effects. Only $4 \%$ of patients indicated that they would rather live out the time they have peacefully, without treatment.

Additional free text comments. Following the multiple choice questions, 15 patients took the opportunity to make further comments. These comments varied from expressing a desire to meet other women in the same situation as themselves to gratitude that healthcare professionals were interested in their opinion, to frustration with lack of support services, out of hours assistance and delays in diagnosis and commencing therapy.

\section{DISCUSSION}

Despite sustained advances in the treatment of locally advanced and metastatic breast cancer over the last few decades, it remains essentially incurable. The 'Being There' survey highlights significant unmet needs among European women and their carers living with metastatic breast cancer. In particular, they want more information regarding their treatment options, including those available in clinical trials. One of the aspects of care that could be improved was also identified as better communication. Nevertheless, for most women, survival was their priority, with them expressing a willingness to 'trade-off the side effects of treatment against prolonging their lives.

This survey serves as a valuable reminder that, for most European women with locally advanced or metastatic breast cancer, survival is their main priority, with most willing to suffer treatment-related toxicities if the treatment will prolong their life. Importantly, patients' views regarding chemotherapy in the palliative setting for all cancers are different from those of a well person or their physicians (Matsuyama et al, 2006; Slevin et al, 1990; Degner and Sloan, 1992), with patients willing to undergo treatments that have relatively small benefits but significant toxicity. Indeed, in one study, $15 \%$ of women who had already undergone treatment for stage I-IIIA breast cancer would choose a high-dose experimental chemotherapy for as little as 1 month of added life expectancy (McQuellon et al, 1995). It is important, however, that patients are sufficiently informed so they can appreciate and understand that a toxic treatment that improves progression-free survival by 1 month does not necessarily equate to an additional month of life.

Over recent years, there has been considerable emphasis on improving communication with patients with cancer. It is of note 

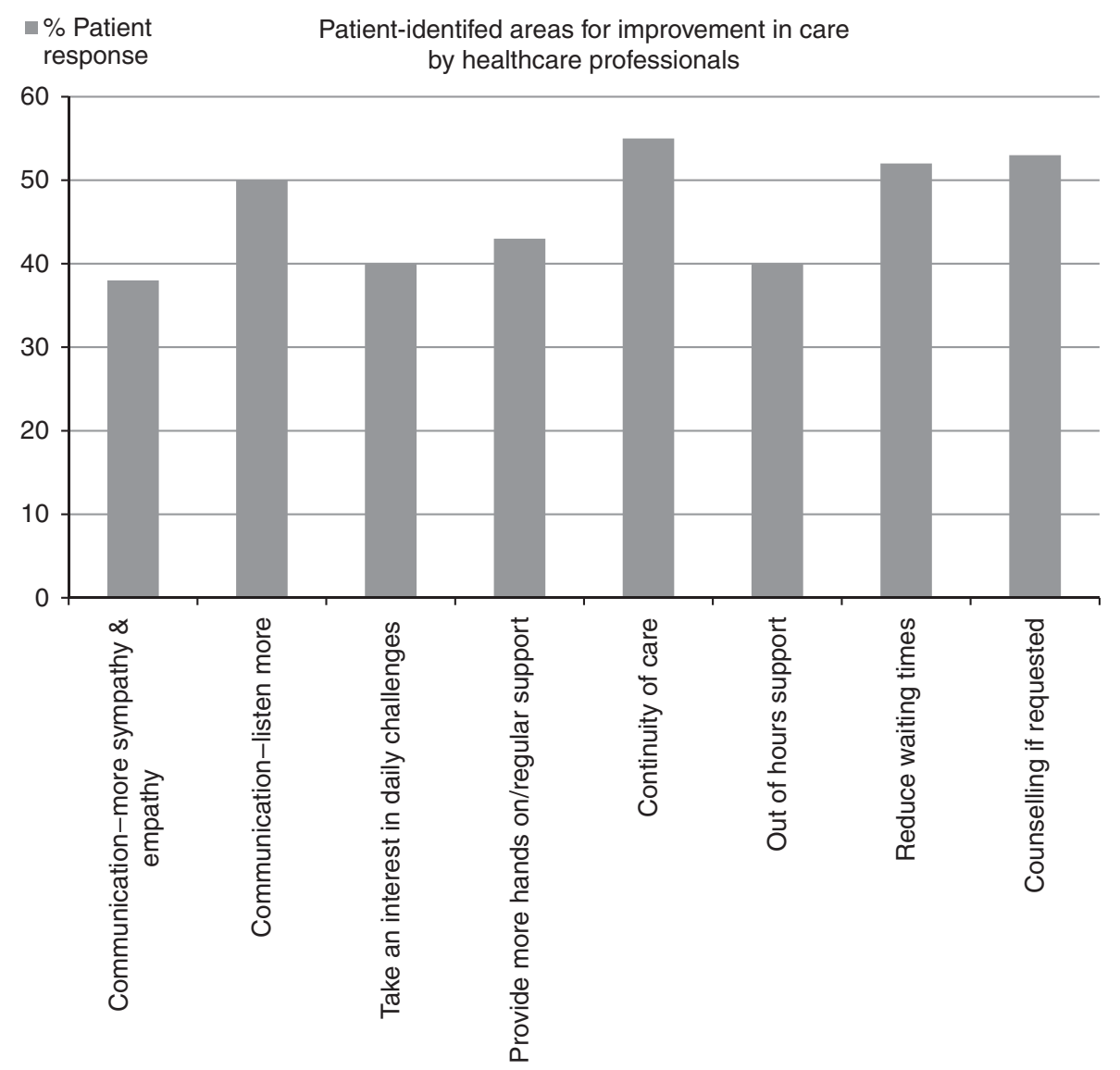

Figure 2. Which areas of care could be improved by healthcare professionals?

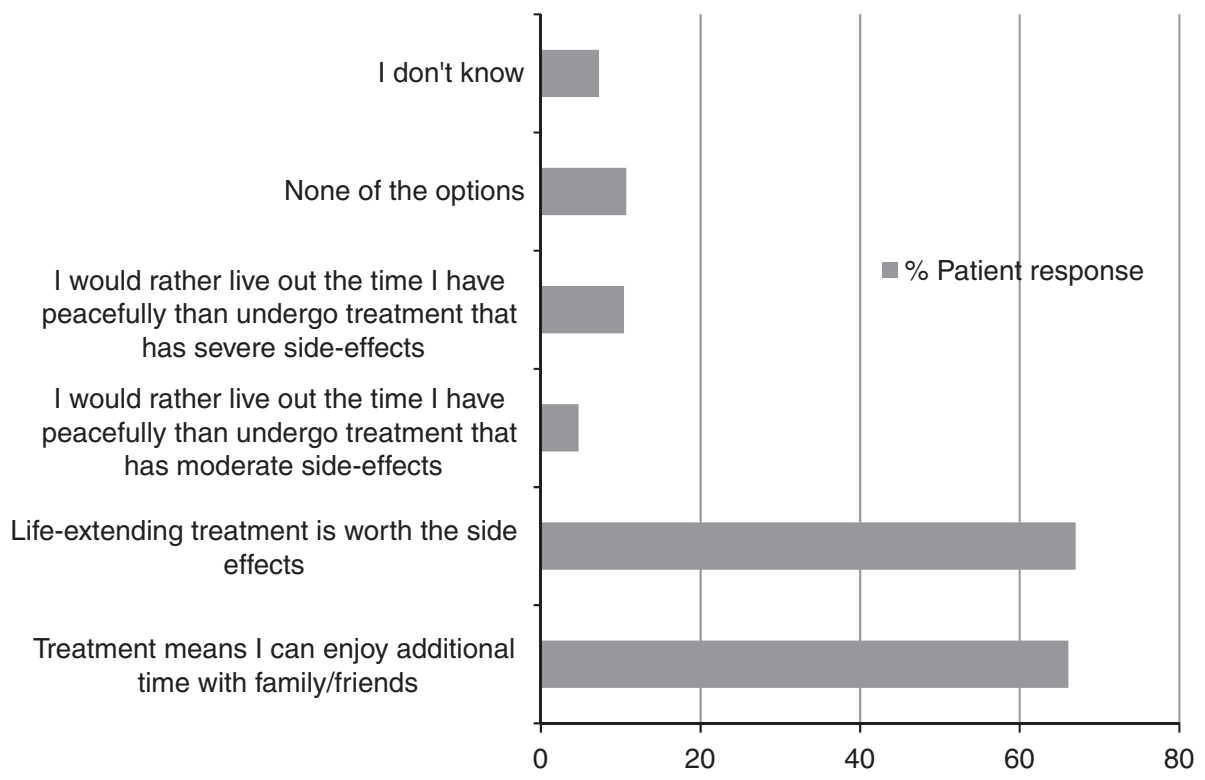

Figure 3. Treatment priorities for women with locally advanced and MBC.

that the women and carers in this survey perceived there to be a lack of information, suggesting current practice may not translate into an optimal patient experience. Furthermore, the survey participants expressed a desire to be more involved in decision making. Treatment preferences for patients with metastatic breast cancer may differ between the woman and her physician due to differing value systems and personal judgements on risk-benefit ratios. Indeed, broad differences exist between different oncologists as to their preferred treatment option (Koedoot et al, 2002), emphasising the importance of the individual patient's preferences and involvement in the decision-making process. Interestingly, despite the increased role of nurses and others in supporting patients with breast cancer, the preference that women receive information from their oncologist noted in the past persists. 
Better information on clinical trials and access to trials was highlighted as a specific priority. Historically, only 5\% of women with breast cancer are enroled into clinical trials (Cohen et al, 2012), with lack of knowledge or adequate information being one of the foremost obstacles to participation (Simon et al, 2004). In the United Kingdom, which has a well-developed National Cancer Research Network, over 2011 to 2012, only $10 \%$ of incidence cases of all breast cancer were enroled into a randomised controlled trial. This survey suggests that collectively we are failing to give our patients the access to clinical trials that they seek. Guidelines from the European Society for Medical Oncology for the management of metastatic breast cancer advise that participation in clinical trials should be offered to all eligible patients, wherever available (Cardoso et al, 2011).

Although many patient support and health groups have developed excellent on-line resources to help patients' access information on clinical trials, several issues are evident. First, because of the rapidity of new trials becoming available, this information is very difficult to keep up to date in any other media than the internet. Hence, patients with little access to the internet are equally likely to be excluded from this repository of information. Second, the amount of information on clinical trials for all cancers is somewhat overwhelming, and accessing appropriate trials may be difficult without a patient knowing her disease in detail. Although the ESMO website has excellent links to nontumour-site-specific websites, most are in English or only a few European languages, and navigating to clinical trials specifically for metastatic breast cancer is not easy. The EuropaDonna.org provides an excellent introduction to clinical trials, although none of the broader support websites have identifiable links for women with advanced breast cancer. Perhaps, both online and paper-based resources could be made available, which are specifically aimed at patients with advanced breast cancer, clearly signposted. The United Kingdom has recently recognised the isolation felt by this cohort of patients, and the Secondary Breast Cancer Awareness Day is now a part of the Breast Cancer Awareness month dedicated to publicising the issues affecting people with secondary breast cancer. We would encourage other countries to adopt such days.

In the current era of personalised medicine, we suggest a more personalised route to information and perhaps the design of specific apps, which can be regularly updated, and even provided in the waiting rooms of clinics, in which patients click on a series of options to guide them to appropriate trials may be an appropriate solution to balancing busy clinics and introducing trials to patients. However, none of this removes the need for discussion of management and care with the patient and carer, and this should include face-to-face discussion of appropriate trials, if the patient and the physician think it to be appropriate.

A worrying one-third of patients and carers in this survey perceived the options available within their countries' healthcare system to be lacking. Moreover, greater than half of the participants were not advised about treatment options available outside of their treatment centre. The wave of 're-centralisation' of healthcare services in many European countries (Saltman, 2008) represents an opportunity for patients to be appraised of a wider range of treatment options in specialist centres, including participation in clinical trials. Despite the apparent shortcomings identified in this study, it is re-assuring that survey participants generally rated their current cancer care as good or excellent.

Inevitably, there are limitations to this study. Although the twopronged design goes some way to tailoring the survey questions to its respondents, the online methodology restricts participation to those with internet access, and hence it is likely to under-represent minorities, older and less well-educated patients, as well as those from lower socioeconomic groups. However, the dissemination of the questionnaire details through the patient support groups was one attempt to open this survey to patients/carers not actively seeking information online, and the multilanguage survey hoped to eradicate the bias for English speakers. Inevitably, the very nature of such a survey is likely to be biased towards those patients actively seeking information, including details of clinical trials, and hence our survey results may over estimate the number of women seeking such information. However, similar results have been identified in the 'Silent Voices' survey (Mayer and Grober, 2006) conducted in the USA (also an online survey) and re-iterate the previously reported isolation felt by women living with advanced breast cancer. The number of responses is also modest, but, being sourced from 17 countries, they do provide a pan-European perspective.

In summary, these findings provide an insight into the unmet needs of women living with locally advanced and metastatic breast cancer in Europe and should be used to improve their services, with an emphasis on improving the provision of information and enhancing patient involvement in decision making.

\section{ACKNOWLEDGEMENTS}

This study was supported by an education grant from Eisai Europe Limited. Results presented in poster format at ESMO, Vienna 2012.

CONFLICT OF INTEREST

CJT is on the speaker's bureau for Eisai. The remaining authors declare no conflict of interest.

\section{REFERENCES}

Bridges JF, Mohamed AF, Finnern HW, Woehl A, Hauber AB (2012) Patients' preferences for treatment outcomes for advanced non-small cell lung cancer: a conjoint analysis. Lung Cancer 77: 224-231.

Cardoso F, Fallowfield L, Costa A, Castiglione M, Senkus E (2011) Locally recurrent or metastatic breast cancer: ESMO Clinical Practice Guidelines for diagnosis, treatment and follow-up. Ann Oncol 22(Suppl 6): vi25-vi30.

Cella DF, Mahon SM, Donovan MI (1990) Cancer recurrence as a traumatic event. Behav Med 16: 15-22.

Chung CT, Carlson RW (2003) Goals and objectives in the management of metastatic breast cancer. Oncologist 8: 514-520.

Cohen E, Belkora J, Tyler J, Schreiner J, Deering MJ, Grama L, Duggan B, Illi J, Pederson J, Anand A, Teng A, Mccreary E, Moore D, Tripathy D, Hogarth M, Lieberman M, Park J, Esserman L (2012) Adoption, acceptability, and accuracy of an online clinical trial matching website for breast cancer. J Med Internet Res 14: e97.

Degner LF, Sloan JA (1992) Decision making during serious illness: what role do patients really want to play? J Clin Epidemiol 45: 941-950.

Ferlay J, Parkin DM, Steliarova-Foucher E (2010) Estimates of cancer incidence and mortality in Europe in 2008. Eur J Cancer 46: 765-781.

Ferlay J, Shin HR, Bray F, Forman D, Mathers C, Parkin DM. GLOBOCAN (2008) v2.0, Cancer Incidence and Mortality Worldwide: IARC CancerBase No. 10. International Agency for Research on Cancer; 2010: Lyon, France. Available from http://globocan.iarc.fr (accessed on 01/03/2013).

Johnston S, Swanton C (eds) (2006) Handbook of Metastatic Breast Cancer. Informa UK Ltd: Oxford.

Koedoot CG, De Haes JC, Heisterkamp SH, Bakker PJ, DE Graeff A, De Haan RJ (2002) Palliative chemotherapy or watchful waiting? A vignettes study among oncologists. J Clin Oncol 20: 3658-3664.

Mahon SM, Cella DF, Donovan MI (1990) Psychosocial adjustment to recurrent cancer. Oncol Nurs Forum 17: 47-52discussion 53-54.

Matsuyama R, Reddy S, Smith TJ (2006) Why do patients choose chemotherapy near the end of life? A review of the perspective of those facing death from cancer. J Clin Oncol 24: 3490-3496. 
Mayer M, Grober SE (2006) 'Silent Voices' Women with Advanced (Metastatic) Breast Cancer share their needs and preferences for information, support and practical resources. Living Beyond Breast Cancer. (available at http://www.lbbc.org/data/news/LBBCsilentvoices.pdf)

Mayer M (2010) Lessons learned from the metastatic breast cancer community. Semin Oncol Nurs 26: 195-202.

McQuellon RP, Muss HB, Hoffman SL, Russell G, Craven B, Yellen SB (1995) Patient preferences for treatment of metastatic breast cancer: a study of women with early-stage breast cancer. J Clin Oncol 13: 858-868.

O'Shaughnessy J (2005) Extending survival with chemotherapy in metastatic breast cancer. Oncologist 10(Suppl 3): 20-29.

Saltman RB (2008) Decentralization, re-centralization and future European health policy. Eur J Public Health 18: 104-106.

Simon MS, Du W, Flaherty L, Philip PA, Lorusso P, Miree C, Smith D, Brown DR (2004) Factors associated with breast cancer clinical trials participation and enrollment at a large academic medical center. J Clin Oncol 22: 2046-2052.
Slevin ML, Stubbs L, Plant HJ, Wilson P, Gregory WM, Armes PJ, Downer SM (1990) Attitudes to chemotherapy: comparing views of patients with cancer with those of doctors, nurses, and general public. $\mathrm{Br}$ Med J 300: 1458-1460.

Wong MK, Mohamed AF, Hauber AB, Yang JC, Liu Z, Rogerio J, Garay CA (2012) Patients rank toxicity against progression free survival in second-line treatment of advanced renal cell carcinoma. J Med Econ 15: 1139-1148.

Wyke A, Barby A, Cowper A, Lilleystone J, Staniszewska S, Williams S (2008) What is 'quality of life' for patients? $\mathrm{Br}$ J Healthcare Management 14: 280-287.

This work is published under the standard license to publish agreement. After 12 months the work will become freely available and the license terms will switch to a Creative Commons AttributionNonCommercial-Share Alike 3.0 Unported License.

Supplementary Information accompanies this paper on British Journal of Cancer website (http://www.nature.com/bjc) 